\title{
Early administration of a probiotic and intestinal HSPs in pig model
}

\author{
M. E. Arnal ${ }^{1}$, M. Formal ${ }^{1}$, A. Cahu ${ }^{1}$, H. Smidt ${ }^{2}$ and J. P. Lallès ${ }^{1}$ \\ ${ }^{1}$ INRA, ADNC, Saint-Gilles, France and ${ }^{2}$ Wageningen University, Wageningen, The Netherlands
}

Maternal or neonatal administration of antibiotics alters offspring intestinal barrier and defense systems in rodents, suggesting an implication of the microbiota ${ }^{(1)}$. The microbiota is suspected to be involved in the onset of metabolic diseases and obesity later in life, possibly through a phenomenon of metabolic imprinting ${ }^{(2)}$. However, specific knowledge on early programming and imprinting of gut function is scarce ${ }^{(3)}$. We have recently shown that in a swine model of peripartum antibiotic treatment, offspring display alterations in various aspects of gut function in the short and (or) long terms ${ }^{(3)}$. The aim of the present study was to test the hypothesis that early probiotic administration to offspring born to antibiotic-treated sows is able to restore some of these altered functions, as already shown in rodents submitted to neonatal stress and treated simultaneously with probiotics.

Gestating sows $(n=23)$ received amoxicillin $(40 \mathrm{mg} / \mathrm{kg} \mathrm{BW} / \mathrm{d})$ orally around parturition (day, d-10 to d21) (EU Interplay project No. 227549, study carried out in 2012 and complying with institutional/national ethical rules). Offspring from 12 litters were given orally Lactobacillus amylovorus DSM 16698 (PROB, $10^{9}$ CFU/pig /dose) at birth and 3 times a week until d 21. Offspring from the other litters $(n=11)$ received the culture medium alone in the same conditions as PROB pigs. Piglets were slaughtered randomly at $\mathrm{d} 14$ and $\mathrm{d} 42$ (short term). The remaining offspring were reared in the same condition until 5 months of age when they continued to be fed the growing diet (LF) or were offered the same diet enriched in fat (HF=LF $+11 \%$ palm oil) for 4 weeks (long term). Homogenous pairs of pigs of a given sex $(1 \mathrm{LF}, 1 \mathrm{HF} ; n=10$ per treatment $)$ within litters were slaughtered. Gut tissues were collected at slaughter and were analysed for villus-crypt architecture by histology and inducible heat shock proteins by western blotting. Data were analysed with SAS using MIXED models.

Probiotic administration did not influence offspring growth performance in the short or long terms nor ileal or colonic villus-crypt architecture in the short term (ongoing analysis for long term). At d14 and d42, HSP27 and HSP70 protein relative levels were lower in the ileum of PROB piglets ( $P<0.01$ and $P<0.05$, respectively; no treatment by time interaction) (ongoing analysis for colon HSPs). In the long term, only HSP27 in the colon was lower in PROB pigs $(P<0.01)$ (no effects of diet or treatment by diet interaction).

To conclude, early oral administration of $L$. amylovorus seems to reduce, according to HSP- and site-dependent patterns, microbiota-induced stress ${ }^{(4)}$ on the ileum (short term) and colon (long term) in offspring born to antibiotic-treated sows and displaying alterations in intestinal barrier and cell protection systems. Although long term effects of the probiotic suggest an imprinting of colonic HSP27, ongoing microbiota analysis will allow us to determine if it is the result of an early microbiota-mediated programming or if probiotic levels and (or) microbiota composition are durably modified by early probiotic administration.

1. Schumann A, Nutten S, Donnicola D et al. (2005) Physiol Genomics 23, 235-245.

2. Rautava S, Luoto R, Salminen S et al. (2012) Nat Rev Gastroenterol Hepatol 9, 565-576.

3. Lallès JP (2012) J. Anim Sci 90(Suppl 4), 421-429.

4. Arvans DL, Vavricka SR, Ren H et al. (2005) Am J Physiol Gastrointest Liver Physiol 288, G696-G704. 